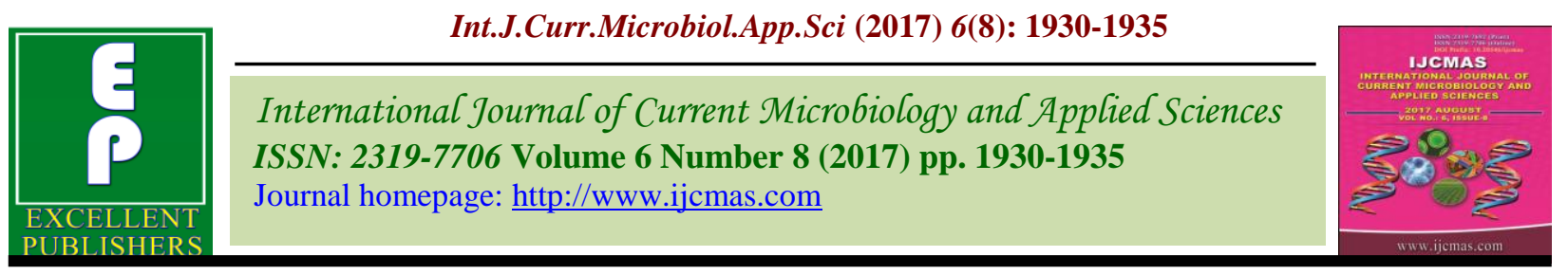

Case Study https://doi.org/10.20546/ijcmas.2017.608.228

\title{
Urosepsis due to Multi Drug Resistant Myroides odoratimimus: A Case Report
}

\author{
Manish Ranjan ${ }^{1}$, Santosh Karade ${ }^{1,2^{*}}$, Praveen Rahi ${ }^{3}$, S.P. Singh ${ }^{1}$ and Sourav Sen ${ }^{1}$ \\ ${ }^{1}$ Department of Microbiology, Armed Forces Medical College, Pune - 411040, India \\ ${ }^{2}$ Department of Pathology, Command Hospital (SC), Pune - 411040, India \\ ${ }^{3}$ National Centre for Microbial Resource, National Centre for Cell Science, Pune - 411021, India \\ *Corresponding author
}

\begin{tabular}{|c|c|}
\hline & A B S T R A C T \\
\hline \multicolumn{2}{|l|}{ Keywords } \\
\hline $\begin{array}{l}\text { Myroides, Multi } \\
\text { drug resistance, } \\
\text { Septicaemia, } \\
\text { Urinary tract } \\
\text { infection. }\end{array}$ & \multirow{4}{*}{$\begin{array}{l}\text { Members of the genus Myroides namely, } M \text {. odoratus and } M \text {. odoratimimus } \\
\text { are ubiquitous in nature. Over past decade, these Gram negative bacteria } \\
\text { are increasingly being implicated in various life threatening infections in } \\
\text { health care setting. Here we report a case of urosepsis due to multi-drug } \\
\text { resistant } M \text {. odoratimimus in a } 41 \text { year old male with anaplastic } \\
\text { astrocytoma. The case highlights the importance of surveillance of } \\
\text { multidrug resistant organisms in intensive care units of hospital. }\end{array}$} \\
\hline Article Info & \\
\hline $\begin{array}{l}\text { Accepted: } \\
\text { 19 June } 2017\end{array}$ & \\
\hline $\begin{array}{l}\text { Available Online: } \\
10 \text { August } 2017\end{array}$ & \\
\hline
\end{tabular}

\section{Case Report}

A 41 year old male; a known case of frontal astrocytoma was brought to this centre with history of headache, altered sensorium and an episode of seizure. He was diagnosed as a case of anaplastic astrocytoma six months back and underwent surgical resection followed by chemotherapy and radiotherapy. Clinical evaluation revealed bedridden but responsive patient with Glasgow coma scale of 6/15. Radiological investigations confirmed recurrence of lesion in right frontal lobe. He was admitted to the intensive care unit (ICU) and underwent emergent surgical resection of the tumor.

The patient had a stormy post-operative course, requiring ventilatory as well as ionotropic support. Histopathological examination of the resected tumor specimen confirmed a grade III anaplastic astrocytoma. On third post-operative day, he developed continuous, moderate to high grade fever with features of hemodynamic instability. Total leucocytes count increased from 11500/cumm on first post-operative day to $16,200 / \mathrm{cumm}$. Peripheral blood smear examination indicated neutrophilia with left shift and toxic granulation. Urine examination showed presence of traces of albumin, reducing substances and numerous pus cells. Blood, urine and surgical drain samples were sent to microbiology laboratory for culture and antibiotic sensitivity testing. 
Urine culture on CLED agar showed nonlactose fermenting, 2-3 $\mathrm{mm}$ dome shaped pale yellow colonies with smooth edge and entire margins (Figure 1a). Gram stained smears from these colonies revealed Gram negative bacilli. The organisms were non-motile and negative for catalase enzyme activity. It was positive for cytochrome oxidase activity. The colonies failed to grow on MacConkey agar, when incubated at $42{ }^{\circ} \mathrm{C}$. This non-fermenter isolate was identified as Myroides sp. by Vitek 2 compact SL (BioMerieux), with good identification score of $94 \%$.

Further identification of the isolate was done using MALDI Biotyper 3.1 (Bruker Daltonics), equipped with autoflex speed and Biotyper database (5597 strains). Mass spectrum was acquired in a linear positive ion extraction mode at a laser frequency of 200 $\mathrm{Hz}$ within a mass range from 2,000 to 20,000 Da (Figure 2). The results of MALDI Biotyper showed the highest (2.47) score value with Myroides odoratimimus LMG 12839, confirming species level identification.

Antibiotic sensitivity testing was performed both by modified Kirby Bauer disk diffusion method and Vitek 2 compact SL (BioMerieux) and interpreted as per CLSI 2016 guidelines (CLSI 2016). Except for minocycline, the isolate was resistant to all available antibiotics recommended for the panel of Pseudomonas sp. The MIC value of tested antibiotics is shown in table 1. Patient, who was initially managed with inj cefoperazone/sulbactum and amikacin was changed to inj meropenem, inj amikacin and tab minocycline. $M$. odoratimimus was also isolated from blood culture by fifth postoperative day. Isolation of similar colonies from both urine and blood cultures confirmed the diagnosis of urosepsis in this case. Our patient continued to remain febrile, developed uraemia, multi organ dysfunction and eventually succumbed to his illness on seventh post-operative day.

Furthermore, we explored for the presence of plasmid families responsible for resistance. The plasmid DNA was extracted using HiPur $A^{\mathrm{TM}}$ Plasmid DNA Miniprep Purification kit as per the manufacturer's instructions and preserved at $-40{ }^{\circ} \mathrm{C}$. We investigated the presence of major plasmid families circulating in the family Enterobacteriaceae as described previously (Carattoli et al., 2005, Carattoli 2009). The primers used for the study were FIA, FIB, FIC, HI1, HI2, I1-I $\gamma$, L/M, N, P, W, $\mathrm{T}, \mathrm{A} / \mathrm{C}, \mathrm{K}, \mathrm{B} / \mathrm{O}, \mathrm{X}, \mathrm{Y}$, Frep B, and FIIA. As both intergenic and intragenic transfer of plasmids are known to confer resistance (Pucci et al., 1988), we used 18 sets of primers divided into 5 multiplex PCRs and 3 simplex PCRs to detect plasmid families (Carattoli et al., 2005). Electrophoresis of the amplified products was performed on $1 \%$ agarose gel and visualized under UV light.

Of the 18 sets of primers tested, we detected presence of a single Frep B plasmid family of amplicon size 270bp in the given isolate (Fig. 3 ). Frep B plasmid has been shown to confer resistance to broad range of beta lactam antibiotics including carbapenems (Huang, Yu et al., 2016). Additionally a commercially available rapid test for detection of carbapenemases production, RAPIDEC® CARBA NP (BioMérieux, France) was performed. Development of orange - yellow colour in this test indicated carbapenemases production in the given isolate of $M$. odoratimimus (Poirel and Nordmann 2015).

\section{Results and Discussion}

The genus Myroides consists of non-motile, oxidase positive, Gram negative bacteria; $M$. odoratus and M. odoratimimus (Garrity et al., 2004). Myroides was taxonomically separated from genus Flavobacterium based on 
genotypic and phenotypic data (Vancanneyt et al., 1996). Although Myroides spp. are ubiquitous, they are frequently encountered in wet environments, sea water, soil and sewage treatment plants. Over past decade, these Gram negative bacteria are increasingly being implicated in various life threatening infections in health care setting (Elantamilan et al., 2015, Endicott-Yazdani et al., 2015). These infections include skin and soft tissue infections, urinary tract infection, bacteraemia and sepsis (Benedetti et al., 2011, Beharrysingh 2017). They have now been increasingly reported as a cause of lifethreatening infections such as, sepsis, urinary tract infection, endocarditis and soft tissue infections primarily in immuno-compromised individuals (Prateek et al., 2015, Beharrysingh 2017). We reported a case of urosepsis due to $M$. odoratimimus in a 41 year old male.

Table.1 Minimum inhibitory concentration (MIC, $\mu \mathrm{g} / \mathrm{ml}$ ) of Myroides odoratimimus for various antimicrobial agents determined by using the VITEK 2 system

\begin{tabular}{|l|l|c|c|}
\hline S. No & Antibiotic tested & MIC & Result \\
\hline 1 & Piperacillin/Tazobactam & $>=128$ & $\mathrm{R}$ \\
\hline 2 & Ceftazidime & $>=64$ & $\mathrm{R}$ \\
\hline 3 & Cefoperazone/Sulbactam & $>=64$ & $\mathrm{R}$ \\
\hline 4 & Cefepime & $>=64$ & $\mathrm{R}$ \\
\hline 5 & Aztreonam & $>=64$ & $\mathrm{R}$ \\
\hline 6 & Doripenem & $>=8$ & $\mathrm{R}$ \\
\hline 7 & Imipenem & $>=16$ & $\mathrm{R}$ \\
\hline 8 & Meropenem & $>=16$ & $\mathrm{R}$ \\
\hline 9 & Amikacin & $>=64$ & $\mathrm{R}$ \\
\hline 10 & Gentamicin & $>=16$ & $\mathrm{R}$ \\
\hline 11 & Ciprofloxacin & $>=4$ & $\mathrm{R}$ \\
\hline 12 & Levofloxacin & $>=8$ & $\mathrm{R}$ \\
\hline 13 & Minocycline & $<=1$ & $\mathrm{~S}$ \\
\hline 14 & Tigecycline & $>=8$ & $\mathrm{R}$ \\
\hline 15 & Colistin & $>=16$ & $\mathrm{R}$ \\
\hline 16 & Trimethoprim/Sulfamethoxazole & $>=320$ & $\mathrm{R}$ \\
\hline
\end{tabular}

Abbreviations:

R - Resistant, S - Susceptible

Fig.1a and 1b 1 (a) shows 2-3 mm dome shaped pale yellow colonies of Myroides odoratimimus on nutrient agar. 1 (b) RAPIDEC ${ }^{\circledR}$ CARBA NP test showing positive result for carbapenemase production indicated by orange yellow colour change in the well e

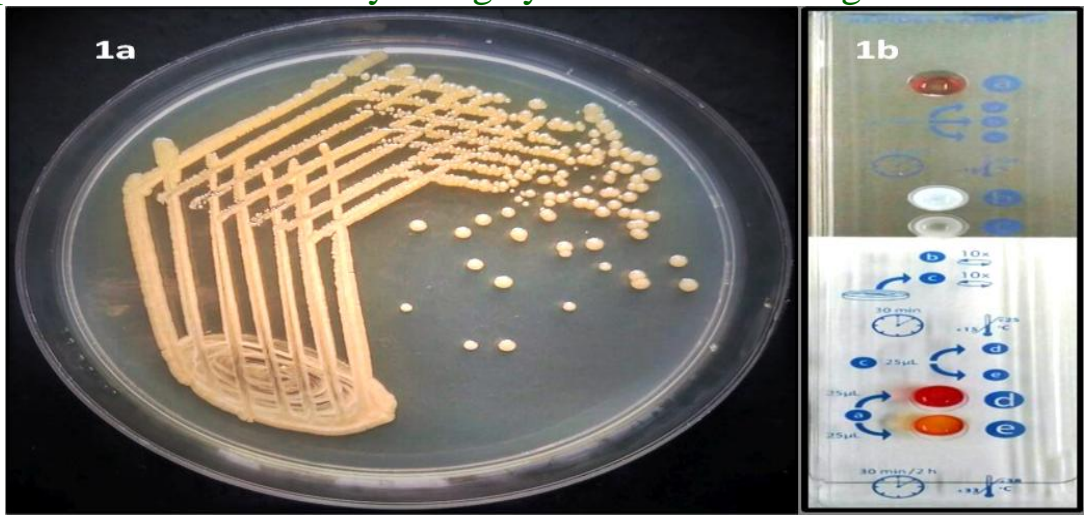


Fig.2 Matrix assisted laser desorption and ionisation, time of flight mass spectrometry (MALDITOF MS) spectrum of whole-cell protein (2-20KDa) urinary isolate of Myroides odoratimimus

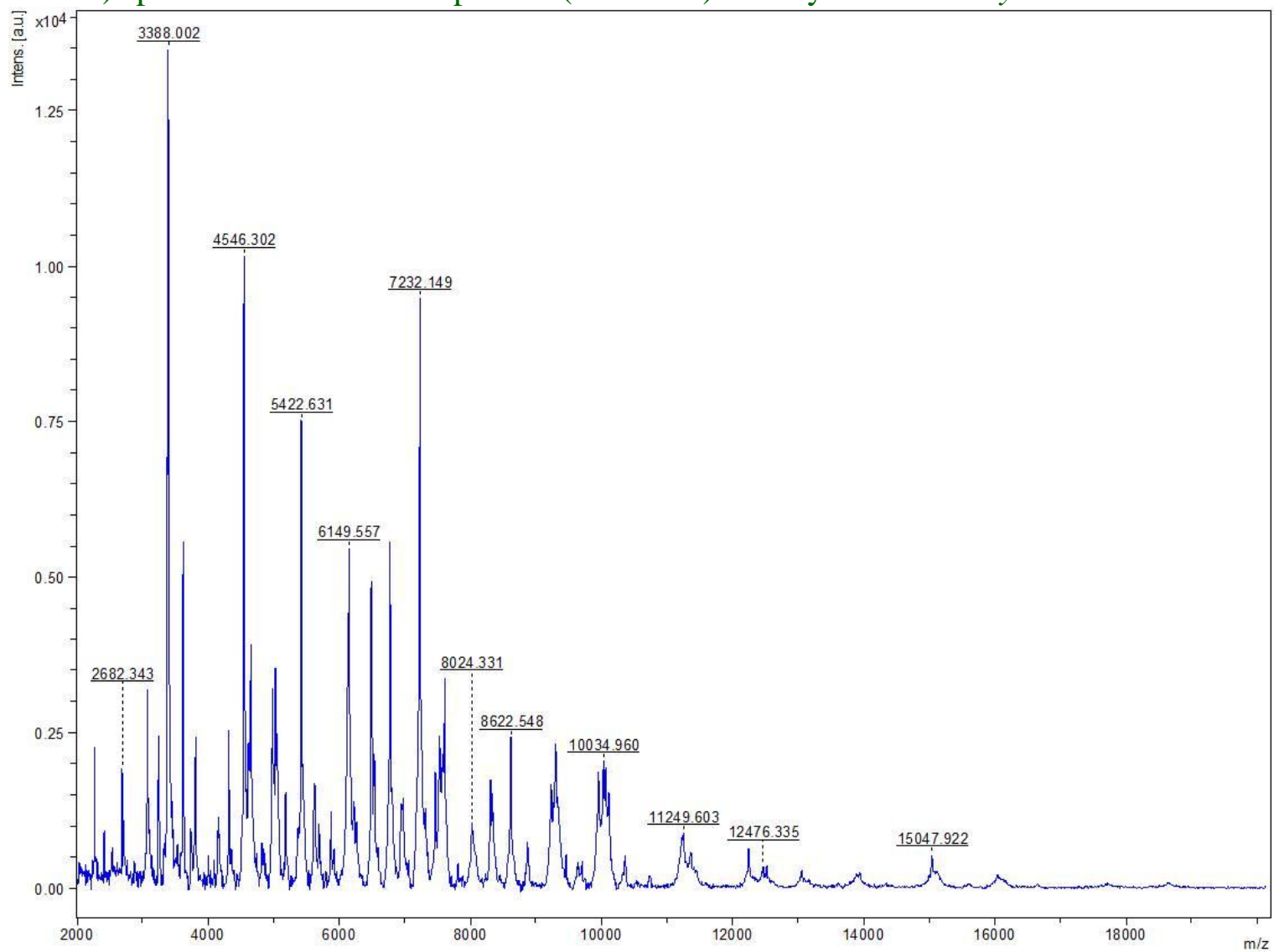

Fig.3 Agarose Gel Electrophoresis of the amplified products of extracted plasmid shows presence of 270 bp FrepB plasmid in lane 1 and 5. Abbreviations: MW - 100bp molecular weight ladder, PC - positive control, NC - negative control

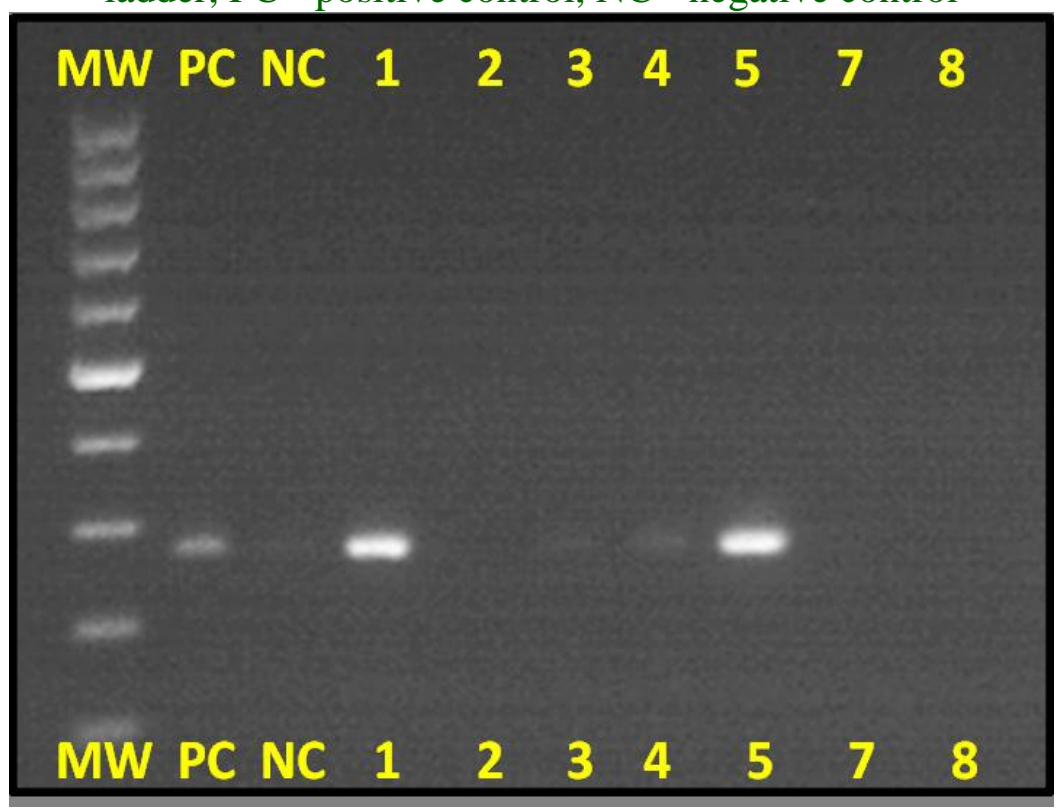


Resistance to multiple antibiotics in Myroides spp, especially intrinsic resistance to betalactam antibiotics has been reported previously (Mammeri et al., 2002, Hu et al., 2016). The present isolate was resistant to all available antibiotics except minocycline. Immuno-compromised status due to ongoing cycles of chemotherapy and radiotherapy, recent surgical intervention, repeated hospital admissions with exposure to multiple antibiotics and prolonged urinary catheterization were important risk factors for acquiring multi-drug resistant Myroides infection in our patient. Although Myroides is commonly found is soil and water, the mode of acquisition in this case could not be ascertained. There was no history of animal bite, injuries or exposure to contaminated water bodies, which is often implicated as source of infection (Maraki, Sarchianaki et al., 2012). Myroides infection are rare in Indian setting and presently only few case reports are published till date (Deepa et al., 2014, Elantamilan et al., 2015, Prateek et al., 2015). Recovery of organism from urine and blood with similar antibiotic sensitivity pattern rules out the possibility of environmental contamination. Also, scrutiny of the recent and past fomite-surveillance data did not reveal recovery of Myroides $s p$ from ICU. The species level confirmation was done by MALDI-TOF which is an accepted tool for identification (Schrottner et al., 2014, Almuzara et al., 2015). However, in spite of extensive antibiotic therapy, the patient succumbed to septicemia and surgical complication. To conclude, this case highlights the role $M$. odoratimimus as an emerging multi drug resistant pathogen in health care settings warranting periodic hospital environmental surveillance to ascertain the source of infection. Use of newer diagnostic modalities is assisting microbiologists not only in rapid diagnosis of exotic infection but also in recommending appropriate antibiotic therapy.

\section{References}

Almuzara, M., C. Barberis, G. Traglia, A. Famiglietti, M. S. Ramirez and C. Vay (2015). Evaluation of matrix-assisted laser desorption ionization-time-offlight mass spectrometry for species identification of nonfermenting Gramnegative bacilli. J Microbiol Methods 112: 24-27.

Beharrysingh, R. (2017). Myroides bacteremia: A case report and concise review. IDCases 8: 34-36.

Benedetti, P., M. Rassu, G. Pavan, A. Sefton and G. Pellizzer (2011). Septic shock, pneumonia, and soft tissue infection due to Myroides odoratimimus: report of a case and review of Myroides infections. Infection 39(2): 161-165.

Carattoli, A. (2009). Resistance plasmid families in Enterobacteriaceae. Antimicrob Agents Chemother 53(6): 2227-2238.

Carattoli, A., A. Bertini, L. Villa, V. Falbo, K. L. Hopkins and E. J. Threlfall (2005). Identification of plasmids by PCRbased replicon typing. $\mathrm{J}$ Microbiol Methods 63(3): 219-228.

CLSI. (2016). M100S, Performance Standardsfor Antimicrobial Susceptibility Testing. 26th edition, Clinical and Laboratory Standards Institute, USA: supplement M100S.

Deepa, R., K. G. Venkatesh, J. D. Parveen, S. T. Banu and G. Jayalakshmi (2014). Myroides odoratus and Chryseobacterium indologenes: two rare isolates in the immunocompromised. Indian $\mathbf{J}$ Med Microbiol 32(3): 327-330.

Elantamilan, D., B. Choudhury, A. B. Khyriem, V. W. Lyngdoh and J. Rajbongshi (2015). Septicaemia caused by Myroides spp.: a case report. JMM Case Reports 2(5). 
Endicott-Yazdani, T. R., N. Dhiman, R. Benavides and C. W. Spak (2015). Myroides odoratimimus bacteremia in a diabetic patient. Proc (Bayl Univ Med Cent) 28(3): 342-343.

Garrity GM, Bell J a, Lilburn TG, Lansing E. Taxonomic outline of the prokaryotes. Bergey's Man Syst Bacteriol. 2004;2(May):1-399.

Hu, S. H., S. X. Yuan, H. Qu, T. Jiang, Y. J. Zhou, M. X. Wang and D. S. Ming (2016). Antibiotic resistance mechanisms of Myroides sp. J Zhejiang Univ Sci B 17(3): 188-199.

Huang, Y., X. Yu, M. Xie, X. Wang, K. Liao, W. Xue, E. W. Chan, R. Zhang and S. Chen (2016). Widespread Dissemination of Carbapenem-Resistant Escherichia coli Sequence Type 167 Strains Harboring blaNDM-5 in Clinical Settings in China. Antimicrob Agents Chemother 60(7): 4364-4368.

Mammeri, H., S. Bellais and P. Nordmann (2002). Chromosome-Encoded Lactamases TUS-1 and MUS-1 from Myroides odoratus and Myroides odoratimimus (Formerly Flavobacterium odoratum), New Members of the Lineage of Molecular Subclass B1 Metalloenzymes. Antimicrobial Agents and Chemotherapy 46(11): 3561-3567.

Maraki, S., E. Sarchianaki and S. Barbagadakis (2012). Myroides odoratimimus soft tissue infection in an immunocompetent child following a pig bite: case report and literature review. Braz J Infect Dis 16(4): 390-392.

Poirel, L. and P. Nordmann (2015). Rapidec Carba NP Test for Rapid Detection of Carbapenemase Producers. J Clin Microbiol 53(9): 3003-3008.

Prateek, S., P. Gupta, G. Mittal and A. K. Singh (2015). Fatal Case of Pericardial Effusion Due to Myroides odoratus: A Rare Case Report. J Clin Diagn Res 9(11): DD01-02.

Pucci, M. J., M. E. Monteschio and C. L. Kemker (1988). Intergeneric and intrageneric conjugal transfer of plasmid-encoded antibiotic resistance determinants in Leuconostoc spp. Appl Environ Microbiol 54(2): 281-287.

Schrottner, P., W. W. Rudolph, B. R. Eing, S. Bertram and F. Gunzer (2014). Comparison of VITEK2, MALDI-TOF $\mathrm{MS}$, and $16 \mathrm{~S}$ rDNA sequencing for identification of Myroides odoratus and Myroides odoratimimus. Diagn Microbiol Infect Dis 79(2): 155-159.

Vancanneyt M, Segers P, Torck U, Hoste B, Bernardet J-F, Vandamme $\mathrm{P}$, et al., Reclassification of Flavobacterium odoratum (Stutzer 1929) strains to a new genus, Myroides, as Myroides odoratus comb. Nov. and Myroides odoratimimus sp. nov. Int $\mathrm{J}$ Syst Bacteriol. 1996; 46:926-32.

\section{How to cite this article:}

Manish Ranjan, Santosh Karade, Praveen Rahi, S.P. Singh and Sourav Sen. 2017. Urosepsis due to Multi Drug Resistant Myroides odoratimimus: A Case Report. Int.J.Curr.Microbiol.App.Sci. 6(8): 1930-1935. doi: https://doi.org/10.20546/ijcmas.2017.608.228 